\title{
Effets nuisibles de la houle et de la marée
}

\author{
RÉSONANCE, MASCARET, ETC., OUVRAGES DE PROTECTION
}

\author{
Harmful effects of wave and tide \\ Resonance, tidal bore, etc., protective structures
}

\author{
PAR D. LAVAL \\ INGĹNIEUR LN GHEF DES PONTS ET CHAUSSÉLS \\ DIRLCTEUR DU PORT DE ROUEN
} rubriques :

Les douze rapports présentés sont regroupés ci-après, en vue de leur examen, sous trois

$1^{\circ}$ Effets de la marée dans les fleuves maritimes;

$2^{\circ}$ Efrets de la houle sur les matériaux;

$3^{\circ}$ Effets de la houle sur les ouvrages.

\section{I. - EFFETS DE LA MAREE DANS LES FLEUVES MARITIMES}

M. Brissavd, dans son rapport sur les divagations et l'ensablement d'un chenal de navigation sous l'effet de la marée, s'efrorce de classer' et d'analyser les phénomènes complexes d'évolution des fonds dans les rivières à marée et les estuaires. Il distingue à cette fin les effets locaux et les effets globaux. Il donne d'autre part quelques indications sur les méthodes d'étude de régularisation des estuaires.

\section{1" Effets locaux;}

M. Buissaun fait un parallèle entre l'action du courant de jusant et celle des basses eaux dans une rivière : les hauts fonds du lit mineur provoquent une augmentation de la pente locale et des vitesses qui facilite l'érosion. L'action du flot se rapproche par contre de celle d'une crue : les inégalités de profondeur du lit mineur ont alors peu d'influence sur les pentes de surface et les vitesses dépendent surtout du rayon bydraulique : il en résulte un surcreusement des mouilles. On constate aussi, dans les rivières à marée, des phénomènes analogues à l'évolution des méandres vers l'aval et aux évolutions cycliques du tracé des chenaux constatés dans les rivières ordinaires.

Ces analogies peuvent servir, dans une certaine mesure, à interpréter les effets de la marée au voisinage de lembouchure ou ils sont prépondérants. M. Brissavo fait ressortir notamment les points suivants.

L'action d'érosion et de transport du flot est prépondérante: le maximum de transport se situe en général au début du flot. Elle s'exerce dans les chenaux profonds. Le flot joue souvent un rôle prédominant dans le régime maritime de l'estuaire : c'est lui qui apporte les sédiments marins arrachés des bancs du large par les courants ou les tempêtes.

Les eftets de la variation périodique des courants sont plus ou moins complexes suivant qu'ils sont simplement alternatifs, suivant une même trajectoire au flot et au jusant on que leur orientation varie à tout instant. Même dans le premier cas, les fosses de flot et de jusant sont rarement en coincidence. Les fosses de flot sont profondes mais courtes (comme les mouilles de crue); les chenaux de jusant sont plus continus mais moins profonds (comme les lits mineurs).

La discordance entre chenaux de flot et de jusant est accentuée lorsque l'orientation des courants varie au cours de la marée. C'est surtout dans un estuaire très large, non canalisé (estuaires en entonnoir par exemple), que cet effet sera sensible. A ce moment, non seulement 
les chenaux ne coincident pas, mais ils sont instables et souvent soumis à des variations eycliques.

\section{$2^{\circ}$ Effets globaux;}

On pent y distinguer des efrets périodiques et des effets non périodiques dus à des variations brusques des conditions naturelles.

Sont à période semi-diurne ou diurne le remaniement du stock sableux, le déplacement du bouchon vaseux; à période mensuelle les recrudescences de transports de matériaux en viveseaux; à périodes plurimensuelles ou annuelles, les effets des tempètes équinoxiales ou saisonnières; à périodes pluriannuelles les évolutions de banc, de chenaux obturés par le développement de barres; à période séculaire les modifications du rivage, les accumulations ou érosions séculaires de matériaux; à très longue période : les évolutions géologiques de subsidence ou d'exhaussement.

Une grande tempête, une marée exceptionnelle peut provoquer des modifications brusques de chenaux. L'équilibre des bancs et des chenaux est toujours précaire et un apport localisé de matériaux peut le bouleverser.

\section{$3^{\circ}$ Méthode moderne d'étude des problèmes de régularisation d'estuaires :}

Elle comporte la recherche systématique des données historiques et géographiques, la multiplication des mesures physiques de couranls, vitesse, turbidité, salinité, etc., l'emploi de modèles réduits. M. Brissaud signale que la méthode de réglage des modèles par la reproduction des évolutions passées a fait d'importants progrès et qu'il a été possible pour la Seine d'obtenir une évolution conforme à la nature pour une période de plus de quatre-vingt-dix ans (1863-1953).

Le rapport de $M$. Brissaud constitue une analyse schématique mais utile des effets de la marée dans les estuaires. Il n'est guère possible, ćtant donnée l'extension du sujet, de reprendre point par point cette analyse. Nous attirerons seulement l'altention sur les questions suivantes :

Le prépondérance des transports de flot est d'autant plus marquée que la dissymétrie de la courbe des marées (raidissement du flot, allongement du jusant) est plus grande. Le courant de flot est alors nettemment plus fort que te courant de jusant si sa durée est sensiblement inférieure. Le dissymétrie est elle-même d'autant. plus forte que l'onde de marée se propage sur des fonds plus faibles: elle s'accentue dans l'estuaire mais peut exister à l'embouchure (cas de la Seine). La sýmétric de l'onde de maréc (Escaut, Tamise) qu'on rencontre en général dans les estuaires profonds est favorable a l'équilibre des actions de flot et de jusant.

De toules les influences signalées, une des plus importantes à l'échelle humaine est la discordance des chenaux de flot et de jusant. Elle apparaît mème dans une partie endiguée, en raison de la diffeulté de réaliser un tracé qui soit satisfaisant à la fois pour les deux sens du courant et permette l'entretien d'un même chenal au flot et au jusant. On sait par exemple qu'un aménagement correct suivant les règles de Fargue conduit à des dispositions des courbes dissymétriques par rapport à leur sommet (diminution de courbure vers l'aval, décalage des points d'inflexion) : ces artifices sont inutilisables pour une rivière à marée. Dans un estuaire libre non endigué, les effets de cette discordance sont encore plus fréquents et plus graves, surtout si des sources abondantes de matériaux mobiles existent à proximité. Le flot s'établit plus tôt et est plus fort dans les fosses ouvertes vers le large, alors qu'il s'établit plus tard et est moins violent dans les chenaux de jusant où ce dernier se fait sentir plus longtemps et qui sont souvent obstrués à l'entrée par une barre sous-marine. Les matériaux apportés par le flot ou enlevés par lui des fosses et des bancs sont ramenés par le jusant dans le chenaux oi il est prédominant et ils y provoquent un développenent continu des barres d'embouchures. Ce processus une fois amorcé se poursuit, en s'aggravant, jusqu'à ce qu'une évolution brusque se produise avec captation du jusant par un chenal nouveau. Une nouvelle évolution cyclique s'ouvre alors.

Ces évolutions sont très bien marquées dans l'estuaire de la Seine suivant un cycle: chenal de jusant sud, chenal central, chenal nord. Leurs inconvénients sont graves : l'instabilité du chenal en rend l'amélioration difficile, un changement de tracé annulant l'efret de tous les travaux effectués.

Les chenaux de rives, surtout si celles-ci sont concaves, sont généralement les plus stables. On peut aider efficacement au maintien d'un tel chenal par des dragages énergiques de la barre d'embouchure : e'est ce qui est fait depuis neuf ans dans le chenal sud de la Seine.

De toute manière, il est rare que dans un estuaire non endigué des améliorations puissent être maintenues sans d'importants dragages.

L'endiguement complet d'un estuaire suivant un calibrage convenable des sections et poussé jusqu'aux grands fonds où l'action érosive des courants est plus faible peut paraître une solution rationnelle de ces problèmes : il est en fait rarement appliqué en raison de son con̂t élevé et des conséquences inacceptables qu'il peut avoir pour le voisinage. On ne peut généralemenl avoir recours qu'à des solutions partielles do 
guidage des courants dont l'étude ne peut être faite utilement que sux modèle.

Bien qu'on n'ait pas encore dégagé, semblet-il, des règles de similitude rigoureuses pour les modèles de fleuves à marée (similitude des profondeurs, des formes de chenaux libres en profil et en plan, des transports de matériaux) et qu'on soit encore obligé d'en déterminer les caractéristiques par des approximations successives basées sur la reproduction de phénomènes naturels connus, il n'est pas contestable que la technique des modèles réduits est d'un grand secours et peut apporter des certitudes si elle est appliquée avec soin.

\section{$*$}

M. BERTHOIS, dans son rapport sur le comportement $d u$ bouchon vaseux en Loire, attire l'attention sur le rôle prépondérant joué par les grands crues dans la constitution du bouchon vaseux. Celui-ci oscille en aval de Nantes suivant une amplitude variable avec le débit propre du fleuve, le débit de marée et la température.

L'amplitude de l'oscillation est, en dehors des périodes de crue, définie par les chiffres suivants :

- En période estivale et par marée moyenne : $40 \mathrm{~km}$. Les eaux turbides n'atteignent ui l'embouchure en aval, ni le port de Nantes en amont;

- En période froide et par marée moyenne : $30 \mathrm{~km}$. Les mises en suspension sont réduites par augmentation de la viscosité de l'eau;

- En période estivale et par grande marée : $65 \mathrm{~km}$. Le port de Nantes est atteint à l'amont au flot; la passe des Charpentiers est atteinte au jusant et une partie de la vase est dispersée au large par les courants marins.

Ces variations sont bien expliquées par celles des courants de marée avec le coefficient et par les modifications de la viscosité de l'eau avec la température qui entraînent une plus grande turbidité en été qu'en hiver.

Mais c'est dans l'étude du régime des crues que l'on doit rechercher une explication de l'origine du bouchon vaseux.

D'après les observations faites au cours des grandes crues de janvier et février 1955 $\left(4.770 \mathrm{~m}^{3} / \mathrm{s}\right)$, M. BerTHors a pu constater que sur 948.000 tonnes de sédiments (dont 730.000 tonnes de vase ou éléments fins) amenés de l'amont au cours de l'année 1955, $86 \%$ ont été apportés pendant les 67 jours de fort débit fluvial. Or, on sait qu'en période de fortes crues, le régime de propagation de la marée est fortement perturbé : sa limite d'action est repoussée de plusieurs dizaines de kilomètres vers l'aval. A la zone de raccordement entre le régime fluvial et le régime de marée on constate de longues étales de courant pouvant atteindre plusieurs heures. C'est dans cette zone que les dépôts apportés par la crue se concentrent et reconstituent le stock de vase qui oscillera ensuite sous forme $d u$ bouchon vaseux tout en s'évacuant progressivement en mer pendant les grandes marées de vives-eaux.

En Loire, c'est dans la région de DongesPaimbœuf que se produit ce phénomène.

Les constatations de M. BERTHOIs donnent une explication très satisfaisante de la constitution $\mathrm{du}$ bouchon vaseux. Elles sont entièrement confirmées par les observations faites en Seine-Maritime après les grandes crues de 1955 : on y a constaté le dépôt dans une section d'environ $6 \mathrm{~km}$ aux environs de Duclair $(25 \mathrm{~km}$ de Rouen) d'à peu près $1.500 .000 \mathrm{~m}^{3}$ de vase. Or c'est précisément dans cette région que se produisent en temps de crue les étales prolongées de courant.

Ce phénomène attire aussi l'attention sur les conséquences nuisibles que pourraient avoir, dans certains cas, des travaux d'aménagement du fleuve augmentant l'influence de l'onde de marée : la zone des dépôts de crue peut être déplacée vers l'amont et atteindre un port qui en était exempt jusque-là. Les envasements des souilles, des quais et des bassins devraient alors être dragués car ils ne peuvent plus être repris par le courant.

$$
\text { 将: }
$$

M. Banal, dans son rapport « Effet du mascaret sur l'amarrage des navires $»$, étudie une action très particulière de la marée dans certains fleuves. Le problème qu'il traite a une grande importance pratique dans la partie aval de la Seine.

Le mascaret se présente en Seine aux plus fortes marées sous forme d'une onde à front assez raide (pente de $2 \%$ et dénivellation de $3 \mathrm{~m}$ à Caudebec; pente de $0,5 \%$, dénivellation de $1,75 \mathrm{~m}$ au Trait à $20 \mathrm{~km}$ en amont de Caudebec). L'étude est faite pour cette dernière localité. L'onde à front raide est suivie par des ondulations dont la période est voisine de $100 \mathrm{~s}$ et l'amplitude de $0,50 \mathrm{~m}$.

M. Banal montre que la force exercée par l'onde frontale sur un navire peut être représentée par un diagramme triangulaire, la force croissant linéairement de 0 à un maximum $F_{0}$ pendant un temps $\mathrm{T}=30 \mathrm{~s}$, puis décroissant jusqu'à 0 pendant un temps égal. La force peut être calculée à chaque instant, avec une approximation suffisante, en supposant une répartition hydrostatique des pressions dans le profil de 
l'onde avançant le long du navire immobile. Toutefois, cet effort doit être majoré d'à peu près $30 \%$ pour tenir compte du fait qu'un corps immergé soumis à un courant accéléré subit une poussée égale à la force nécessaire pour donner la mème accélération à la masse d'eau qu'il déplace augmentée d'une masse fictive complémentaire (elle est d'à peu près $30 \%$ pour un navire de formes courantes). L'effort maximum $F_{0}$ peut être ainsi évalué à $120 \mathrm{t}$ pour un navire déplaçant $15.000 \mathrm{t}$.

M. BANAL étudie ensuite le comportement des amarres. Il admet que celles-ci ne développent aucun effort tant que le déplacement du navire est inférieur à une certaine valeur $x_{0}$ (rattrapage du mou), puis que l'effort est ensuite proportionnel au déplacement et égal à $\mathrm{K}\left(x-x_{0}\right)$.

Le calcul de l'effort des amarres pour un navire de masse fictive totale $M$ donne des résultats très différents suivant leux état de tension initial.

Si le mou est suffisant pour que la tension des amarres n'apparaisse qu'après disparition de la force extérieure, ce qui se traduit par la condition $x_{0}>r=\mathrm{T}^{2} \mathrm{~F}_{0} / \mathrm{M}$, la tension maximum atteint $\mathrm{C}=\mathrm{F}_{0} \mathrm{~T} / \theta$ avec $\theta=\sqrt{\mathrm{M} / \mathrm{K}}$. Il $\mathrm{y}$ a alors intérêt à utiliser des amarres très extensibles (K petit).

$\mathrm{Si}$ le mou des amarres est nul, $x_{0}=0$, et l'amarrage parfaitement élastıque, l'effort $\mathrm{C}$ est toujours inférieur à $1,52 \times \mathrm{F}_{0}$.

Enfin, dans les cas intermédiaires, $0<x_{0}<r$, l'effort dépend de $\mathrm{T} / 9$ et $x_{0} / r$ : il est donné par' un graphique. C'est le cas de la pratique.

Le graphique montre alors l'intérêt d'employer des amarres pré-tendues et très extensibles. Il montre le danger d'amarrages rigides présentant un jeu important.

Des essais sur modèle réduit ont confirmé le mode de caleul de la poussée. Mais ils onl montré que le phénomène était compliqué par les réactions entre navire et quai dues à l'obliquité des amarres. Des essais en vraie grandeur avec amarrages rigides constitués par des poutres métalliques ont été effectués sur un chaland pesant $550 \mathrm{t}$. Des enregistrements au straingauge ont fait apparaitre des efforts très élerés et de très courtes durées au passage des ondulation suivant le front raide: leur analyse n'est pas terminée.

Il est certain que l'étude de M. Banal montre bien le sens des phénomènes; ses résultats rejoignent d'ailleurs ceux de l'étude de l'amarrage des navires soumis aux oscillations de longue période du port du Cap. Mais son étude théorique doit être complétée par des mesures en vraie grandeur comme celles qui sont actuellement entreprises.

\section{II. - EFFETS DE LA HOULE SUR LES MATERIAUX}

L'étude de M. LARRAS, « Effets de la houle et du clapotis sur les fonds de sable », est d'une grande importance théorique et pratique. M. Larras s'est proposé, à l'aide d'études en laboratoire, de rechercher les lois de l'érosion des sables sous l'action de la houle et du clapotis.

Ses études ont porté sur des matériaux de densité et de granulométrie très variées soumis aux effets d'un clapotis de $6,00 \mathrm{~m}$ de longueur d'onde, de $2,01 \mathrm{~s}$ de période et d'amplitude variant entre $0,10 \mathrm{~m}$ et $0,27 \mathrm{~m}$. Il a également essayé ces mêmes matériaux sous forme d'un talus à pente faible $(1 / 6)$ soumis à l'action de houles monochromatiques de périodes $2 \mathrm{~s}, 4 \mathrm{~s}$ et $6 \mathrm{~s}$. Il a successivement déterminé : les vitesses sur le fond de début d'érosion, les lois de formation de fosses d'érosion créées par un obstacle de saillie déterminée placé dans le clapotis au ventre des vitesses, les rides parasites (ripplemarks):

\section{SEuILS D'ÉROSION :}

Les mouvements de matériaux commencent lorsque la vitesse maxima $V_{m}$ sur le fond au cours d'une période atteint la valeur :

$$
V_{m}=9,5 \frac{(\delta-1)^{1 / 3}}{T^{1 / 2}}+W(\text { en } \mathrm{cm} / \mathrm{s})
$$

où W est la vitesse de chute libre des grains de matériaux dans l'eau, o leur densité, $T$ la période du mouvement.

Fait assez remarquable, la différence $V_{n}-W$ ne dépend pas du diamc̀tre des grains. M. Larras signale cependant que ce résultat n'est bien vérifié que pour le cas, effectivement réalisé dans la nature, où le diamètre des sables n'atteint pas l'épaisseur de la couche limite qui serait pour la houle et le clapotis de $1,3 \sqrt{v T}$ où $v$ est le coefficient de viscosité cinématique. Cette épaisseur varierait de $\varepsilon=2,9 \mathrm{~mm}$ pour $\mathrm{T}=5 \mathrm{~s}$ à $\varepsilon=5 \mathrm{~mm}$ pour $\mathrm{T}=15 \mathrm{~s}$. Sans vouloir attacher trop d'importance à cette remarque, nous ne pensons pas qu'on puisse affirmer que l'épaisseur de la couche limite des houles naturelles est celle donnée par une telle formule. Les idées sur la formation de la couche limite en mouvement alternatif et notamment dans le cas où la turbulence existe depuis la surface jus- 
qu'au fond (1) sont encore peu claires. La quantité $\varepsilon=1,3 \sqrt{\vee T}$ est l'épaisseur de la couche a vitesse rapidement variable au voisinage du cond dans le cas du mouvement laminaire d'un lluide visqueux de profondeur constante $h$, soumis à une force alternative de période $T$ : elle ne parait pas directement applicable an cas de la houle turbulente (2).

Une autre conséquence remarquable de ta formule est que, pour des périodes croissantes, $\gamma_{m}$ se rapproche de la vitesse de chute $W$ et ceci lautant plus que le diamètre est important. Par exemple, pour un sable siliceux de $1,15 \mathrm{~mm}$, une houle de période $12 \mathrm{~s}$, on a approximativement : $W=9,5 \mathrm{~cm} / \mathrm{s} ; V_{m}-W=3 \mathrm{~cm} / \mathrm{s}$

On notera enfin la petitesse relative des vitesses limites d'entraînement sur le fond (moins de $10 \mathrm{~cm} / \mathrm{s}$ pour des sables siliceux de $1 \mathrm{~mm}$ et des houles de l'ordre de $10 \mathrm{~s}$ ). De telles vitesses sont atteintes pour des clapotis on des houles de très faible amplitude.

\section{ProfOndEURS D'ÉROSION :}

M. LanRas a trouvé que la profondeur limite d'érosion de part et d'autre d'un obstacle de saillie donnée, placé an ventre des vitesses du clapotis, ne dépendait, toutes choses égales d'ailleurs, que de la densité des matériaux à l'exclusion de leur diamètre et de leur granulométric. Il paraît en déduire, sans toutefois l'expliciter, que les fosses d'érosion sont à l'échelle dans le modele lorsqu'on utilise des sables de même densité que dans la nature quoique de diamètre différent.

Mais, par ailleurs, on doit multiplier les profondeurs limites d'érosion, lorsque, toutes choses ćtant égales d'ailleurs (c'est-ả-dire pour une même profondeur et une même période), on passe d'un matériau de densité $\delta$ à un matériau de densité $\delta^{\prime}$ et d'un clapotis de creux $h_{c m}$ a un clapotis de creux $h_{\text {om }}^{\prime}$ par un coefficient :

$$
\mathrm{K}=\log \left[\left(\frac{h^{\prime}}{10}\right)^{3, \mathrm{j}} \frac{1}{\delta^{\prime}-1}\right]: \log \left[\left(\frac{h}{10}\right)^{3.5} \frac{1}{\delta-1}\right]
$$

L'intervention de la quantité $1 / \delta$ - 1 signific que l'érosion n'a pas de limite si $\delta^{\prime}=1$. L'intervention de la quantité $\left(h^{\prime} / 10\right)^{3,5}$ est plus imprévue. Elle parait traduire le fait que pour une hauteur du clapotis inférieure ou égale à celle donnée par :

$$
\left(\frac{h^{\prime}}{10}\right)^{3.5} \frac{1}{\delta^{\prime}-1}=1
$$

(1) Ce qui se produirait pour le cas généralement réalisé dans la pratique où la profondeur $H<0,3(2 L)$. Sur tontes ces questions voir: Mrche, Propriétés des trains d'ondes océaniques.

(2) Voir aussi l'étude de M. VALEMBors (question III). les vitesses $V$ sur le fond deviennent trop faibles pour provoquer un affouillement. II est surprenant que la période n'intervienne pas, le rapport $V / h$ en dépendant fortement. Il est à remarquer que l'étude a été faite pour une période de clapotis constante é égale à $2,01 \mathrm{~s}$.

Il serait probablement utile, tant pour vérifier la similitude annoncéc des fosses d'érosion que pour apprécier l'influence de la période, que les essais soient poursuivis à des échelles et pour des périodes variées.

\section{DUREE D'ÉROSION :}

L'érosion naît au voisinage du ventre des vitesses où la vitesse maxima est $\mathrm{V}_{0}$; elle se développe jusqu'au point qui correspond à la vitesse de seuil d'érosion $V_{m}$. Le temps qu'elle met pour atteindre le point où la vitesse maxima est $\left(V_{0}-V_{m}\right) / 2$ a été trouvé égal à $\theta=6(\delta-1)^{2}$ minutes. M. Larras ne précise pas si cette formule est valable seulement pour $T=2,01 \mathrm{~s}$ ou pour loule valeur de T. L'extension des essais proposée ci-dessus semblerait également utile pour vérifier ce point.

Les essais de M. LARRAs ouvrent la voie à des études qui s'avéreront certainement fécondes sur les lois jusqu'ici inconnues de la similitude des transports de matériaux par des courants alternatifs.

$$
\text { ** }
$$

MM. Vincent et Gamot, dans leur « Etude sur la défense des ports maritimes contre lensablement $»$, examinent plus particulièrement le problème d'un port établi sur une côte soumise à un transit littoral sous l'effet de houles obliques. Ils cherchent à établir les conditions optima de fonctionnement du système de protection par pompage des sables en amont du port et refoulement en aval. Ils comparent ensuite l'économie de ce procédé et de celui qui consiste à allonger la jetée de protection du port au fur et à mesure de l'ensablement.

En ce qui concerne les dispositions techniques, MM. VIncent et Gamot concluent à la supériorité d'une station de pompage mobile sur une station de pompage fixe. La station mobile se déplacerait sur une estacade parallèle à la jetée du port et à une assez grande distance en amont. La pompe peut alors entretenir un sillon perpendiculaire au rivage qui est alimenté par les transports de houle, non seulement de l'amont vers l'aval, mais encore de l'aval vers l'amont, c'est-à-dire de la jetée du port vers l'estacade. Dans le cas où par grande tempête le pompasse est impuissant à compenser les apports, ceux-i viennent s'accumuler contre la jetée et peuvent être repris ultérieurement.

Ces dispositions paraissent judicieuses, mais posent un certain nombre de questions. 
Quelle doit être la longueur de l'estacade portant la pompe mobile? Il semble a priori qu'elle doive être du même ordre de grandeur que celle de la jetée à protéger. Il est signalé en effet d'après les études sur modèle que le transport peut se produire jusqu’à une ligne où la profondeur est de quatre fois l'amplitude de la houle au large. Bien que les mouvements soient surtout localisés au voisinage du déferlement, il faut que la pompe puisse avancer à grande distance de la côte pour éviter d'être contournée par le large. La construction et l'entretien d'une passerelle exposée à la houle par grande profondeur peuvent être très coûteux. Quelles sont d'autre part les dispositions pratiques et quel est le prix du pompage? Des élindes traînantes du type de celles employées sur les dragues suceuses en marche peuvent probablement convenir, mais quelle expérience a-t-on de leur emploi dans une mer houleuse presque en permanence comme on les rencontre souvent sur les côtes à fort transit de sable?

Les auteurs indiquent que pour le port de Macuripe, au Brésil, le système du pompage serait nettement plus économique que l'allongement des jetées. On doit semble-t-il se garder de généraliser une telle conclusion et apporter une grande prudence dans les calculs économiques en raison de l'absence d'une expérience prolongée des systèmes de pompage envisagés.

M. Chatel, dans son rapport : «Quelques aspects de la défense des côtes en France. Enseignements à tirer d'une visite générale du littoral », apporte les enseignements d'une visite générale du littoral français organisée par le Comité d'Océanographie et d'Etudes des Côtes. Après un rappel des phénomènes naturels qui pourraient expliquer l'aggravation des érosions littorales constatée depuis le début du siècle (élévation du niveau relatif de la mer, augmentation de la fréquence des tempêtes constatée notamment sur les côtes anglaises') l'auteur indique les causes imputables à l'intervention de l'homme. On ne peut que souscrire sans réserve aux remarques qu'il présente sur l'influence néfaste des prélèvements de matériaux sur les plages, des ouvrages trop raides et empiétant sur l'estran, du manque d'entretien. Il observe que si beaucoup d'ouvrages longitudinaux s'avèrent dangereux ou nuisibles, les épis paraissent au contraire avoir réussi presque partout. Enfin, il évoque les aspeets administratifs et techniques de la défense des côtes : la législation francaise, en laissant celle-ci à la charge et à l'initiative des riverains (sauf subvention de l'Etat), est certainement peu favorable à une action d'ensemble méthodique et suivie.

\section{III. - EFFETS DE LA HOULE SUR LES OUVRAGES}

L'étude de M. BaudelaIre, sur \&l'agitation d̀ longue période dans le port d'Alger $\gg$, apporte une nouvelle preuve du fait que l'agitation que l'on qualifiait autrefois dans certains ports du terme assez vague de ressac est souvent l'effet de phénomènes de résonance de longue période. La suite des constatations faites à Alger est particulièrement instructive. On a commencé par observer des mouvements de va-et-vient de tlotteurs d'une amplitude de $40 \mathrm{~m}$ et de $90 \mathrm{~s}$ de période. On en a déduit que ces déplacements pouvaient être les signes de l'existence de seiches dont ils sont en effet des conséquences caractéristiques. On a alors procédé sur un modèle réduit à l'échelle du $1 / 150$ à des expériences d'excitation sous l'effet de trains de houle modulés suivant des périodes variant entre $45 \mathrm{~s}$ et 4 ou 5 minutes et on a noté les périodes de résonance, la position des nœuds et des ventres d'oscillation. Puis on s'est efforcé de vérifier dans la réalité les observations faites sur modèles, à l'aide d'un appareil enregistreur très mobile extrapolé d'un appareil de laboratoire et basé sur les variations d'une capacité électrique. On a trouvé une cö̈ncidence très satisfaisante faisant apparaître suivant les bassins des seiches de $70 \mathrm{~s}$ à $240 \mathrm{~s}$. Les ingénieurs du port d'Alger cherchent actuellement à établir une relation entre l'état de la mer au large et l'agitation dans le port à l'aide de mesures des houles : ils envisagent même de construire un modèle spécial portant sur l'ensemble des côtes algéroises.

On ne peut que souhaiter la continuation de telles études, l'utilisation parallèle du modèle réduit et des mesures dans la nature étant seule susceptible d'apporter une explication de l'origine des oscillations de longue période dans les ports.

$$
\text { *** }
$$

M. Hensen, dans son mémoire relatif aux «Efforts des houles sur les ouvrages 》, rend compte de deux séries d'études qu'il a entreprises sur l'effet des houles sur un môle vertical. Dans une première partie, il examine la variation du coefficient de réflexion $\mathrm{H}_{\mathrm{R}} / \mathrm{H}_{\mathrm{A}}\left(\mathrm{H}_{\mathbb{R}}\right.$ hauteur de la houle réfléchie, $H_{\Lambda}$ hauteur de la houle incidente ou suivant les notations habituelles en France $2 h_{\mathrm{R}} / 2 h_{\mathrm{A}}$ ) en fonction de deux variables: la cambrure $\gamma=\mathrm{H} / \mathrm{L} \quad(\gamma=2 h / 2 \mathrm{~L}$ suivant nos 
notations) et le rapport de la profondeur devant l'ouvrage à la demi-longueur d'onde $\mathrm{K}=2 \mathrm{~h} / \mathrm{L}$ lou $K=H / L$ avec nos notations). Dans une deuxième partie, il analyse les résultats de 124 enregistrements d'efforts mesurés sur la digue de Naples et les compare aux efforts calculés.

On peut être surpris de voir consacrer une tude au coefficient de réflexion de la houle devant une paroi verticale : on peut croire qu'il est toujours égal à l'unité. Mais en réalité la réflexion n'est parfaite que si la houle incidente cl le clapotis formé n'ont subi aucun déferlement dovant l'ouvrage.

On admet en général que cette condition est rialisee si les trois relations suivantes sont respectées:

$$
\begin{gathered}
\gamma<0,140 \text { th } \pi \mathrm{K}, \quad \mathrm{H}>5 \mathrm{~h} ; \\
\mathrm{K}=\mathrm{H} / \mathrm{L}>2 / 7=0,28
\end{gathered}
$$

(avec les notations habituelles). Mais en dehors de ces limites, des destructions d'énergie peuvent se produire et le coefficient de réflexion être, par suite, inférieur à l'unité.

l.es conditions de stabilité données ci-dessus peuvent se traduire avec les paramètres de M. HENSEN: $\gamma<0,140$ th $\pi \mathrm{K} ; \gamma<\mathrm{K} / 5$; $\mathrm{K}>0,28$; la première condition est plus sévère que la seconde pour $K>0,7$ et vice versa pour $K<0,7$.

Les résullats donnés par M. Hensen sont bien dans le sens attendu : mais ils different notablement dans le détail des règles énoncées.

$1^{\circ}$ Il semble bien que pour $K<0,28$ il $y$ ait une instabilité de toutes les ondes, sauf celles de très faible cambrure : $\gamma<1 / 100$;

$2^{\circ} \mathrm{Si} K>0,28$ et $\gamma>0,01$, le coefficient de rúflexion $t$ dépend de $\mathrm{K}$;

$f$ n'est pratiquement égal à 1 que pour $\gamma<0,05$ et $\mathrm{K}>0,60$, sinon il est toujour's inférieur à 1 ; $3^{\circ}$ Il $\mathrm{y}$ a toujours déferlement pour $\gamma \geqslant 0,10$;

$4^{\circ}$ Les causes de la réflexion incomplète seraient différentes suivant que $\gamma<0,055$ ou $\eta>0,055$. Dans le premier cas, londe résultante n'atteint pas le double de l'onde incidente parce que sa forme est modifiée par insuffisance des profondeurs. Dans le deuxième cas, il y a perte d'énergie par déferlement.

$5^{\circ}$ Les conditions nécessaires à la formation d'un clapotis régulier devant une digue verticale seraient donc assez nettemment plus sévères que celles tirées de considérations théoriques.

Ces résultats sont à rapprocher de ceux obtenus expérimentalement par M. DANEL (Gravity Waves, 1951) (1). Celui-ci a trouvé également que le clapotis était instable si la cambrure de l'onde incidente $\gamma$ est $>0,10$. Par contre, ses

\footnotetext{
(1) Et également des essais sur modèles de la digue verticale de Marseille décrits dans le rapport de M. Cov. TEAUD analysé plus loin.
}

essais vérifiaient de manière satisfaisante la relation $\gamma<\mathrm{K} / 5$, pour $\mathrm{K}<0,50$. D'autre part il a obtenu des clapotis stables pour

$$
0,10<\mathrm{K}<0,28 \text {. }
$$

Une comparaison des processus expérimenLaux suivis serait utile. II apparait en tout cas que les risques de déferlement du clapotis soient très sérieux, même par grande profondeur, dès que $\gamma \geqslant 0,10$.

La seconde partie de la note est consacrée à la comparaison entre les résultats des mesures de pression faites à Naples et du calcul conduit suivant la méthode dite hydrostatique (pression due à un clapotis régulier). Bien que la dispersion des résultats soit considérable, il apparaît que pour les ondes de grande amplitude la divergence entre la théorie et les mesures est faible et que la théorie donne des efforts plus grands que la mesure. Par contre, pour les ondes de faible amplitude les écarts sont considérables $(300 \%)$, tantôt dans un sens, tantôt dans l'autre. Constatation intéressante qui confirme la sécurité du calcul pour les grandes ondes, mais incite à la prudence pour l'appréciation des effets des houles de faible amplitude.

M. Lhermitte, dans sa \& Contribution it l'étude de la stabilité des ouvrages de protection maritime $»$, introduit une notion nouvelle pour apprécier la probabilité de ruine sous l'effet de la houle d'un ouvrage pendant une période déterminée (20 ans par exemple). Il remarque en effet qu'il existe entre l'amplitude d'une houle et le temps pendant lequel elle doit agir pour ruiner un ouvrage une relation qu'on peut essayer de déterminer sur modèle. Il propose donc d'apprécier la probabilité de ruine d'une digue en recherchant pour une période sans entretien la probabilité d'existence d'une houle d'amplitude minima donnée pendant un temps correspondant à celui qui est nécessaire pour que cette amplitude entraine la ruine de louvrage.

En supposant une répartition gaussienne des périodes de temps durant lesquelles sévit une houle d'amplitude minima donnée, il montre que la probabilité cherchée pour une durée d'action $t$ est :

$$
q(t)=(1 / a) e^{-t / a}
$$

oì $a$ est la probabilité de présence de la houle considérée, donnée par les statistiques de houle.

La probabilité cumulée, c'est-à-dire la probabilité d'observer une houle donnée pendant un temps $t$ supérieur ou égal à un temps donné $t_{0}$ est :

En prenant $t_{0}=18 \mathrm{~h}$ et une période sans entretien de un an, il trouve que la probabilité 
de ruine pour un ouvrage calculé pour la houle décennale (probabilité de présence de 1/3.650) est élevée (1/100 en 20 ans; $2,5 / 100$ en 50 ans). Elle n'est plus respectivement que de $2 \cdot 10^{-8}$ et $5 \cdot 10^{-8}$ pour la houle trentenaire.

Bien que basée sur des lois de probabilité théoriques qui ne sont peut-être pas applicables exactement à la houle, l'étude de M. Lhermitte n'en est pas moins très intéressante dans son principe et ouvre une voie nouvelle dans l'appréciation de la sécurité des ouvrages maritimes.

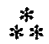

M. Cavenel, dans sa « Note sur les dégâts causés da la digue verticale de Gênes par la tempête des $19 / 20$ février $1955 »$, procède à une étude approfondie de la cause de ces dégâts. On ne saurait que souscrire entièrement à ses conclusions.

Il montre que pour une houle d'amplitude $2 \mathrm{~h}=7,00 \mathrm{~m}$, comme celle qui s'est produite, la digue a dû supporter une lame déversante de près de $3,00 \mathrm{~m}$ et que les assises supérieures do la digue Galliera ne pouvaient pas résister au glissement à la pression hydrostatique calculée suivant les règles habituelles, compte tenu de la surélévation du niveau moyen; quant aux sections de digues en caissons alvéolaires, elles ont péri par destruction des parois des alvéoles.

M. Cayenel fait ressortir une coïncidence vraiment remarquable entre l'implantation des brèches dans le tronçon est de la digue et les points de pression maxima donnés par M. LARRas dans son étude de 1952 (Travaux). L'influence de la diffraction de la houle au voisinage du musoir paraît donc bien confirmée par l'expérience. L'auteur signale que la même répartition n'apparait pas cependant dans la partie centrale : ce fait est peut-être dû à l'éloignement des musoirs et il ne ferait alors que confirmer la théorie. Une remarque très importante est celle faite sur linfluence de la longueur d'onde sur la stabilité du talus de soubassement.

\section{**}

M. J. Couteaud présente un « Rapport sur la digne verticale de Marseille ».

Il est important de noter que la digue de Marseille a été déterminée pour une houle de $7,00 \mathrm{~m}$, bien qu'on n'ait jamais observé de houle supérieure à $5,50 \mathrm{~m}$. Cette majoration de $30 \%$, pour empirique quelle soit, traduit bien la difficulté de prévoir les plus grandes houles possibles sur une longue période d'existence d'un ouvrage.

Au nombre des indications précieuses données sur la stabilité de l'ouvage, on notera:
-- La nécessité de surveiller particulièrement les talus de fondation et les renforcements successifs qui ont été apportẻs it ces derniers (blocs de $4 \mathrm{t}$ au lieu de 1,5 $\mathrm{t}$; approfondissement de la muraille verlicale; jointoiement des blocs de talus par du bitume coulé sous l'eau).

- La nécessité de réaliser une fondation par. faite des blocs et d'armer le bloc de répartition inférieur.

-- L'intérêt de réaliser entre piles de blocs une liaison plastique, par exemple avec du mortier bitumeux pour éviter les oscillations entre piles.

Un autre point d'un haut intérêt pratique est la différence des frais d'entretien d'une digue à talus et d'une digue verticale. Bien que les frais d'entretien d'une digue verticale soient seulement la moitié de ceux d'une digue à talus, on doit remarquer qu'en valeur absolue la différence est faible $(1.500 \mathrm{~F}$ par mètre linéaire et par an pour des ouvrages coûtant plusieurs millions par mètre linéaire). C'est donc seulement lorsqu'on est sûr de la stabilité d'une digue verticale, comme l'expérience semble le permettre à Marseille, qu'on doit avoir recours à ce type d'ouvrage.

M. LE Gorgeu expose, dans l'étude « Action de la houle sur les portes d'écluses du port de Dunkerque $»$, un problème pratique très impor. tant, celui de la manœuvre des portes d'écluses exposées à la houle. La solution adoptée pour l'écluse Trystram est extrêmement robuste puisqu'elle doit permettre d'encaisser des efforts pendant la manœuvre de $240 \mathrm{t}$ sur un vantail. Des limiteurs d'efforts doivent permettre d'ap. précier, en exploitation, les efforts réellement subis. Leur observation sera très précieuse pour fixer les règles expérimentales à suivre en matière de portes d'écluse.

La note montre également combien sont fra giles à la houle, surtout pendant leur manœuvre, les portes roulantes comme celles de l'écluse Watier. Des portes à deux vantaux comme celles adoptées pour l'écluse Trystram sont préférables; les portes à secteur comme celles de Dieppe ou de Saint-Malo sont probablement encore mieux adaptées au problème.

\section{ADDITIF AU RAPPORT GENERAL}

Nous examinons ci-après deux communications parvenues après l'établissement du présent rapport général.

L'étude de M. Bovrnières sur les Oscillations de longue période à 'Tamatave est à rapprocher' 
de celle de M. Batdelarre sur l'agitation dans le port d'Alger. On a relevé à Tamatave des oseillations de $30 \mathrm{~s}$ dans les darses de batelage, de $90 \mathrm{~s}$ dans la darse aux long-courriers et nêne de 8 minutes et demie sur les appareils enregistreurs placés dans les darses de batelage. M. Bourniéres rappelle que l'étude de tels phénomènes et des remèdes à leur apporter trouve sa justification dans les inconvénients qu'ils présentent pour les navires : il peut se produire une résonance entre la période propre d'oscillations du navire amarré et la période de la seiche. Les calculs faits par M. Wilson pour le Cap ont montré que la période propre du navire pouvait varier entre $10 \mathrm{~s}$ pour un navire de $8000 \mathrm{t}$ amarré serré et $120 \mathrm{~s}$ pour un navire de 45000 t amarrè lâche.

Le problème de Tamatave a été étudié sur modèle réduit. L'auteur examine les difficultés de reproduction des phénomènes : le réglage de la rugosilé, des coefficients d'absorption et de réflexion est très délicat. Il insiste sur le problème de la perméabilité des digues aux ondes Iongues dont les lois de similitude, compte tenu de la forte distorsion (4) introduite dans le modèle, sont encore mal connues.

Les essais ont montré que des mesures partielles comme la création à l'intérieur de la rade d'obstacles réfléchissants pouvaient être satisfaisantes pour certaines périodes et certaines zones du port mais très dangereuses au contraire pour d'autres périodes. Il faut des travaux importants, tels que la fermeture complète de la grande passe de Tamatave, pour modifier les caractéristiques des bassins résonants et obtenir une amélioration certaine. Il faut, d'autre part, réaliser des digues particulièrement étanches, les ouvrages en enrochements exécutés sans soins particuliers étant très perméables aux ondes longues.

L'étude faite pour Tamatave n'a pas abordé le problème de l'origine des seiches. Les phénoménes de résonance de la rade ont été étudiés sur modẻle sous l'effet d'ondes monochromatiques et non de trains de houle. Il est possible que l'origine même des seiches ait une influence non négligeable sur l'efficacité des mesures prises : par exemple sur la perméabilité des ouvrages.

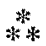

MM. LE PORT et BASTARd, en exposant laccidenl survenu à la digue de Lesconil et les mesures prises pour réparer l'ouvrage, attirent l'attention sur les problemes particuliers aux digues dites du type « mixte », employées dans les mers à marée et constituées par une muraille verticale fondée au-dessus des basses-mers sur un talus d'enrochement. La muraille doit résister aux effets de la houle déferlante sur le talus, effets qui d'après les études de BAGNold et d'IrIbarren seraient représentés approximativement par un diagramme rariant linéairement de 0 au niveau de $1,5 \mathrm{~h}$ au-dessus de la crête de houle à $8 \mathrm{~h}$ au niveau du creux de la houle, et prenant des valeurs rapidement décroissantes au-dessous ( $2 \mathrm{~h}$ hauteur totale de la houle au moment du déferlement). Les pressions qu'exerce la houle déferlante sur les parties superieures d'un ouvrage sont donc beaucoup plus fortes que celles d'un clapotis régulier ( $2 \mathrm{~h}$ au niveau moyen et au-dessous). Ce sont d'ailleurs ces surpressions qui sont probablement à l'origine des dégâts constatés sur certaines digues verticales fondées par profondeur insuffisante et dont le couronnement est emporté par les effets du déferlement.

Cependant, en Bretagne, des ouvrages de ce type ont bien résisté avec des superstructures alvéolaires particulièrement légères, conçues par Consinkre, lorsqu'ils étaient établis par de faibles profondeurs voisines du niveau de basse mer. La houle ayant déferlé longtemps avant d'atteindre la muraille avait perdu alors une grande partie de sa force vive.

L'exemple de Lesconil où l'ouvrage était établi par des fonds plus importants, descendant jusqu'à $(-5,80)$ montre qu'une digue mixte est alors très exposée. La superstructure a été attaquée à la fois par déversement de la houle audessus de la muraille avec érosion du talus intérieur et par ripage vers le port sous l'effet des pressions.

On a étudiè sur modèle réduit deux profils pour la reconstruction : l'un en digue mixte tròs renforcée; l'autre en digue à talus de faible pente $2 / 1$ et arasée à $1 \mathrm{~m}$ seulement au-dessus du niveau de haute mer. Ce dernier profil s'est révélé plus résistant, sans être plus coûteux.

\section{LES '́NERGIES DE LA MER}

Compte rendu intégral des QUATRIEMES JOURNÉES DE L'HYDRAULIQUE. de la Société Hydrotechnique de France

DeuX volumes, brochés.......... $8000 \mathrm{~F}$ 


\title{
DISCUSSION
}

\author{
A. - SEANCE DU 15 JUIN, MATIN \\ (Président: M. A UBERT)
}

M. le Président remercie les participants présents à Ia séance et rend hommage à l'esprit scientifique des rapporteurs qui, dans une question se rattachant plutôt à l'art de l'ingénicur, se sont élevés au-dessus du niveau de la technique.
Il propose d'ordonner la discussion suivant les divisions du rapport général et, après la lecture de chaque partie de ce rapport par M. LAVAL, donne la parole aux personnes désirant intervenir sur cette partie.

\section{I. - MAREE DANS LES FLEUVES MARITIMES}

M. Lacombe attire l'attention sur une question de terminologie suggérée par la lecture du rapport général (p. 2, colonne de gauche, en bas) et relative à la nécessité de distinguer nettement les étales de courant et les étales de niveau, les phases du courant et les phases des variations de niveau. Le Service Hydrographique de la Marine emploie dorénavant, conformément à un usage ancien, les termes de « montant» et « perdant» pour les variations de niveau et les termes de « flot 》 et « jusant» pour les phases du courant de marée (supposé alternatif ou approximativement alternatif). Ainsi des confusions et de nombreuses controverses seront évitées.

M. Laval se déclare entièrement d'accord avec M. LACombe sur cette question de terminologie.

M. Berthois demande à M. Brissaud s'il a fait des mesures lui permettant de contrôler l'origine marine des sédiments de l'estuaire de la Seine. Les faits ordinairement invoqués comme la présence, dans les sédiments estuariens, de minéraux lourds, de petits graviers patinés par l'hydroxyde de fer ou de manganèse, ou bien de diatomées, de même que la teneur en carbonate de chaux. ne sont pas significatives de l'origine marine, car on retrouve les unes ou les autres de ces caractéristiques dans les cours moyens de certains fleuves : même assemblage de minéraux lourds et teneur en carbonate de chaux dans les limons et la craie du bassin de Paris; graviers patinés par l'hydroxyde de fer et diatomées dans la Loire, au-delà de Saumur.

D'autre part, les travaux récents de JenLov (1951) Pardé (1952-1953), Lafond (1953), Van Ander et Postma (1954), Berthois (1955) (1), qui ont montré l'importance des apports fluviaux et l'origine continentale des sédiments estuariens de nombreux cours d'eau, conduisent à penser que les estuaires envahis par des sédiments d'origine marine sont en nombre restreint.

M. BRissaud répond que l'origine marine des sédiments de l'estuaire de la Seine a été, dès 1788 , prouvée par Lamblardie père en démontrant par le calcul que ces sédiments ne pouvaient être attribués ni au recul des falaises du Calvados, ni à la destruction des galets; cette origine marine est également confirmée par la comparaison des volumes de dépôts sableux - et non vaseux - qui, d'après VolMat, atteignent 5 millions de mètres cubes par an derrière les digues avec, d'une part les débits de marée qui peuvent atteindre $500.000 \mathrm{~m}^{8} / \mathrm{s}$, d'autre part les débits de crue qui ne dépassent guère

(1) 1951 : Tellus, vol. 3, pp. 123-127.

1952-53: Rv. Géogr. Alpine, t. 39, pp. 5-40; t. 47, pp. 399-420

$1952-53$ : Rv. Gégr. Alpine, t. 39, pp. 5-40;
1953 : Bull. Soc. Gél. Fr., t. 3, pp. $175-189$.

1953 : Bull. Soc. Géol, Fr, t. 3, pp. 175-189.

1954 : North. Hol. Pub. Comp., vol. 1 , pp. $1-2$
1955 : C. Rend. Ac. Sc., t. 241 , pp. 231-232.
$2.000 \mathrm{~m}^{3} / \mathrm{s}$. Enfin, l'influence des travaux d'avancement des ouvrages sur les rolumes des dépôts sableux, visible sur les graphiques qui se trouvent dans le rapport de M. BRISSAUd et notamment, la discontinuité qui apparaît immédiatement après les premiers travaux (1840-1850) montrent bien l'origine marine de ces dépôts apportés par le flot et retenus par les digues au moment du jusant. Par ailleurs, le barrage de Poses retient une partie des sédiments fluviaux qui sont d'ailleurs faibles dans la partie amont de la Seine.

A son tour, M. Laval prend la parole afin de confirmer que pour l'estuaire de la Seine les cubatures qu ont été effectuées sont suffisamment démonstratives de l'origine marine des sédiments. En même temps que le dépôt de plusieurs centaines de millions de mètres cubes derrière les digues, il $\mathrm{y}$ a un amaigrissement corrélatif des bancs du large. M. LAVAL fait d'ailleurs remarquer à M. BERThols que les profondeurs moyennes des bancs sont beaucoup plus importantes dans la Loire que dans la Seine. Dans la Seine il se produit un vérilable décapage des bancs situés à la cote de basse mer, et, à certaines heures de la marée, l'eau est chargée, jusqu'à 9 à $10 \mathrm{~g}$ par litre, de matériaux. L'origgine des phénomènes cycliques mentionnès dans le rapport de M Laval se trouve dans les dépôts considérables partout où le jusant s'épanouit. D'autre part, la Seine n'apporte rien; pendant $99 \%$ du temps les debits solides d'amont sont nuls, et les seuls apports lors des grandes crues sont dragués à leur arrivée dans le port de Rouen et sont exclusivement constitués de vase.

M. Laval reconnaît toutefois qu'après l'intervention de M. Benthors il ne faudrait pas généraliser les conclusions de M. Brissaun. Il y a beaucoup de fleuves, en particulier ceux à estuaire profond, où les phénomènes d'envasement ou d'ensablement sont probablement d'origine amont. M. Laval conclut en faisant remarquer qu'il n'y a pas contradiction entre les idées de M. Brissaud et celles de M. Berthors. Il faut simplement les replacer dans leur cadre véritable, e'est-à-dire, que charpue estuaire pose son problème et nécessite une solution particulière après une étude approfondie.

M. Gantel souligne la réelle fidélité du modèle à fond mobile, étudié par le Laboratoire Dauphinois d'Hydraulique et par MM. Laval et BANAL, qui a permis de reconstituer en 3 mois l'histoire de la Seine depuis 90 ans, au point de vue topographique et hydrographique, en particulier l'engraissement du Bec du Ratier.

M. LaRras signale que le problème de l'amarrage des navires dans le mascaret présente de grandes analogies avec celui de l'amarrage dans les seiches. D'où la possibilité de compléter les indications de M. Banal par 
d’utres, toutes nouvelles, issues d'études récentes sur las seiches:

I" Non-proportionualité probable des forces de rappel des amarres aux déplacements (influence du poids et de la tension du câble);

y* Difficulté de maintien des très grands navires à poste fixe le long des quais, quelle que soit la raideur des amarres, par suite de leur obliquité excessive vis-à-vis de l'horizontale et de la direction générale du navire.

il. Laval répond que les approximations faites à ce sujet par M. Banal lui paraissent valables dans le cas de lamarrage en Seine en raison de la place dont on dispose et qui permet une faible obliquité des amarres par rapport à lhorizontale et à la direction du navire.

M. Larras apporte les compléments suivants aux indications, qui datent de quelques mois, de son rapport :

1. Les essais effectués avec un liquide glycériné ont confirmé les prévisions du rapport quant à linfluence quantitative de la viscosité.

2. Les essais effectués tout dernièrement sur l'entraîminent du sable par les houles à couche limite turbnlente ont permis d'étendre la validité de la formule proposée dans le paragraphe II nettement au-dela du cas, retenu seulement jusque-là, des houles à couche limite laminaire.

3. La formule $6(\delta-1)^{2}$ ne vaut, comme indique dans le rapport, que pour les houles de période 2 secondes, de sorle que le coefficient 6 dépend peut-être de la période, mais il faut en retenil surtout que la densité du sable constitue l'élément essentiel de l'échelle des temps.

4. La formule de transposition des profondeurs d'érosion qui figure dans le rapport vaut très certainement d'un essai sur modèle a l'autre, mais il est encore trop tot pour l'appliquer dans le cas de très grands changements d'échelle comme c'est le cas entre nature et laboratoire. Tout an plus peut-on la combiner avec l'hypothèse, à vérifier, qu'il y aurait similitude de Froude des profondeurs d'érosion s'il y avait similitude de Froude des dimensions du modèle et des dimensions du sable.

5. Les indications du rapport (dont il n'est pas question dans le rapport général) sur le problème des rides donnent le moyen de séparer les rides d'oscillation de l'imase géométrique des fosses d'ćrosion grâes à des changements d'échelle qui les affectent différemment; les rides disparaissent pour des matériaux très lourds ou très léger's, leur longuear d'onde est proportionnelle à lexcursion des molécules (rapport voisin de 0,80 ).

Après une remarque élogieuse de M. le Président sur la précision des résultats obtenus par M. Larras dans un probleme nouveau, $M$. Laval, sur la demande de M. Dieulor, donne des précisions sur les appareils qui permettent l'étude, dans la nature, de la mise en mouvement des sédiments :

1" Le début de monvement est décolé par les appareils qui permettent de répéter les sons comme ceux qui ont été construits par M. REmenienas et ceux qui ont été utilisés en Loire-Maritime.

2" Le transport peut être déterminé par la méthode des prélèvements d'eau ou au moyen d'intégrateurs de dépôts de transport, ou capteurs, appareils laissés en place et dans resquels le sable s'accumule.

Ces appareils donnent des résultats sensiblement exacts pour les transports en suspension mais sans doute moins conformes à la réalité pour les mouvements de fond car les capteurs placés sur le fond troublent le mouvement.

Ayant été cité par le rapporteur au sujet des mesures des transports solides, M. SIegraned croit utile de founnlr quelques précisions.

Pour le charriage en saltation des sables des estuaires, des prélèvements sur le fond n'ont pas à sa connaissance donné de mesures valables. Un appareil a été mis au point par un ingénieur du Service, M. Junier, qui avait pour but de déceler acoustiquement le dibut du mouvement sur le fond. Pour des mesures de débit solide sur le fond, on a cherché à observer le comblement de sillons transversaux, parfois spécialement crés à cet effet ou encore réalisés à l'occasion de poses de canalisations sous-marines; encore fau-il noter dans les sections fluvio-maritimes que le charriage résiduel (offet jusant + flot) n'est pas donné par le comblement du sillon sur ses parois amont $t$ aval. Enfin, l'observation des déformations et du mouvement résiduel des riddens dans le fond ridé de la Loire a permis des estimations, rapportées par M. BaLlade dans une communieation il y a 3 ans.

Pour le transport en suspension, des prélèvements permettent l'étude des taux de suspension ou turbidités. que l'on rapproche ensuite des vitesses et débits aux mêmes points. Ciest sur leurs bases que M. Berthors a calculé dans sá communication le transport solide en suspension apporté par la Loire fluviale dans sa section lluvio-maritime (il s'agit d'un calcul et non a'un « constat » comme le dit le rapporteur général). C'est sur les mêmes bases qu'est étudié le bouchon vaseux fluvio-maritime, dont la connaissance est particulitrement importante pour suivre lentretien de la section et étudier ses conditions d'encombrement; d'où l'intérêt. des recherches poursuivies par M. Bentrous avec le Service Maritime de la Loire-Inférieure.

M. Benthois a d'ailleurs indiqué les tonnages de sédiments fins apportés en un an, et noté d'autre part l'apport en suspension d'éléments sableux pendant les crues, tous évalués cn tonnes de sédiments secs. Il s'y ajoute évidemment le transport des mêmes sables en charriage (en génćral évalué en mètres cubes), et c'ent l'ensemble qui forme l'apport solide d'amont dans la section fluvio-maritime.

M. Siegrien désire faire remarquer à ce sujet combien les dépôts diffèrent de volume, à égalité de matière apportée, selon qu'il s'agit d'éléments fins ou grossiers : une tonne de matière sèehe sable donne 6001 environ de sable en place, tandis qu'une tonne de sédiments fins secs donne un volume de dépôt vaseux de deux fois et demi à trois fois plus élevé.

D'où l'intérêt des chiffres donnés par M. Bentrors et plus largement des études de l'apporl solide d'amont, puisque le problème de l'encombrement progressil de la section fluvio-maritime de la Loire est capital pour son avenir.

M. Schr.f fait une potite lemarque sur la question posée par M. Dieulot :

Il $y$ a eu pendant une période de 30 ans ou plus un développement intensif en plusieurs pays d'appareils pour la mesure du débit solide dans les fleuves et les estuaires, aussi bien sous la forme de charriage, que de saltation et de suspension. Ce développement a abouti a un certain nombre d'appareils qui ne donnent peutêtre pas une précision absolue, mais avec lesquels on a eu certainement une expérience pratique satisfaisante et très utile. On pourra trouver sur ce sujet dans la litterature internationale une documentation abondante.

M. le Président remercie $M$. SchiJ de sa remarque intéressante; il estime toutefois, comme M. Siegrarien l'a constaté, que les appareils ou méthodes de mesure du débit solide ne donnent guère d'indications suffisamment prëcises, sauf peut-être, dans le cas ou un fleuve déversant et décantant entièrement dans un lac, comme le Rhòne au Léman, permet une intégration précise par la mesure géométrique de son delta et par des prélevements destinés à la mesure de densité. 


\section{II. - AGITATION DE SEIGHES DANS LES PORTS}

M. Bourriéres désirerait des informations complémentaires sur un point du rapport de M. Baudelaire. Il y est cité un déplacement horizontal de $40 \mathrm{~m}$ au poste Sète IV dont la darse oscille en quart d'onde. II serait important de connaître la profondeur d'eau et l'amplitude verticale des oscillations.

A Tamatave, en effet, pour une darse semblable, oscillant en quart d'onde, avec une profondeur de $8 \mathrm{~m}$ on a trouvé, pour une période de 90 secondes, un rapport entre oscillations verticales et horizontales inférieur à 10. Si les données sont comparables à Alger, $40 \mathrm{~m}$ horizontaux correspondraient à plus de $4 \mathrm{~m}$ verticaux.

M. Baudelaire répond à la première question de M. Bournières que la profondeur au poste de Sète IV varie de $4 \mathrm{~m}$ en fond de darse à $5,75 \mathrm{~m}$ environ à son origine.

En ce qui concerne les déplacements, M. Sineyjol, ancien chef d'exploitation au port d'Alger, indique que les déplacements verticaux en fond de darse étaient de l'ordre de $1 \mathrm{~m}$ et que les déplacements horizontaux de l'ordre de $40 \mathrm{~m}$, indiqués, n'étaient probablement pas généraux sur toute l'ouverture de la darse, ce qui expliquerait la distorsion que l'on ne retrouve pas ailleurs.

M. LhenMTte signale que les enregistrements ont mis en évidence au port de la Pointe des Galets (à la Réunion) l'existence d'une seiche de 90 à $100 \mathrm{~s}$ de période. Ce chiffre est à rapprocher de celui cité par M. Bourrières pour Tamatave $(90 \mathrm{~s})$. Le problème de l'excitation de ces mouvements se pose donc, et l'on peut se demander s'il existe dans cette zone de l'Océan Indien des mouvements ondulatoires de cette période.

Les expériences sur modèle ont montré, toutefois, que le mode de résonance dans le cas du port $t_{1}$ de la Pointe des Galets était très particulier : l'établissement de la seiche n'est possible que par suite de la résonance existant entre les périodes de l'avant-port et du port qui sont très voisines.

\section{III. - EFFORT DE LA HOULE SUR LES OUVRAGES}

M. Dhaille a remarqué, au cours d'essais faits à la demande de l'administration italienne, qu'à Gênes, sur le prolongement Est du môle Galliera, les ruptures se sont produites en des points qui correspondent bien aux points de renforcement dus à la présence du musoir. Ceci confirme les indications de M. Cavenel. M. Dhaille ajoute qu'en dehors des causes signalees par M. CaveNEL il y a certainement eu, à Gênes, non seulement un clapotis très important, mais aussi un déferlement avec gifles sur la paroi verticale.

M. DE Rouvilue signale quelques-uns des résultats avancés à la toute récente réunion du Comité des Efforts des Lames (A.I.P.C.N.) à Bruxelles.

Les Italiens ont constaté un relèvement du niveau moyen de la mer et, par suite, un relèrement de la pression exercée sur une digue verticale dans la partic haute, compensée, il est vrai, par une légère réduction des pressions dans la partie basse par l'obliquité de la ligne enveloppe correspondante, relèvements beaucoup plus considérables qu'on ne l'avait retenu aux Congrès de Navigation de Bruxelles (1935) d'abord et de Rome (1953) ensuitc.

On peut se demander si, sur une digue verticale, même suffisante en pied dans l'eau, mais couronnant un très haut massif d'enrochements, s'étalant par suite assez loin avant par de grandes profondeurs, il ne s'exerce pas un déferlement partiel amorcé par ce massif.

M. Laval confirme l'intérêt et la valeur des mesures italiennes contrôlées par des mesures photogrammétriques sur les répartitions des pressions le long de la digue de Naples : on peut en déduire que le relèvement du niveau moyen devant une digue excède celui qu'on constate dans la houle beaucoup plus largement que les calculs théoriques actuels ne l'indiquent; M. Iribarren a suggéré que ce relèvement anormal semble dû au fait qu'une demi-longueur d'onde de la houle est engagée sur le talus en enrochement qui supporte, sur une assez grande épaisseur, la digue de Naples, d'où une modification de profil (simple hypothèse).

M. Miche précise que, cette digue reposant sur un talus de grande profondeur assez raide, ceci pourrait être une première cause, au moins partielle, des fortes surélévations observées du niveau moyen (jusqu’a $2 / 3 \times 2 h$, semble-t-il, si $2 h$ est l'amplitude de la houle incidente). Il résulte en effet d'études théoriques (GovnRET et l'auteur), que l'amplitude du clapotis produit sur un talus à $45^{\circ}$ est plus que doublée par rapport à celle de la houle incidente; elle vaudrait $4 h \sqrt{\overline{2}}$ au premier ordre d'approximation et la surélévation du niveau moyen devrait être accrue de ce fait. Toutefois, ceci concerne les houles longues; pour des houles courtes, la digue de Naples devrait fonctionner comme une jetée verticale habituelle, et cette cause d'accroissement ne devrait plus exister.

D'autre part, la surélévation du niveau moyen (cote moyenne entre celle de l'affleurement maximum et minimum sur la digue, comparée à la cote du niveau de repos) ne représente pas une quote-part fixe de l'ampli. tude incidente $2 h$, mais croit comme $h^{2}$. Autrement dit, la quote-part précédente augmente avec la cambrure de la houle, qui doit être précisée pour interpréter correctement la surélévation produite.

Au surplus, il n'est peut-être pas indispensable, pour expliquer de fortes surélévations, de prendre en compte l'effet de talus. Lorsqu'on considère les termes du second ordre de la houle, la surélévation obtenue est à peu près double de celle calculée par Saintlou. Gette surélévation théorique semble assez approchée pour de fortes cambrures, même pour des houles limites sur le point de déferler. De toute façon, avant de pouvoir apprécier pleinement les observations faites à Naples, des précisions supplémentaires sont nécessaires.

M. le Président remercie tous les rapporteurs et les orateurs qui ont participé à la discussion, et notam. ment, M. LavaL, Rapporteur Général.

\section{B. - SEANCE DU 15 JUIN, APRES-MIDI \\ (Président : M. Larias)}

\section{III. - EFFORT DE LA HOULE SUR LES OUVRAGES (suite)}

Apres ayoir remercié M. Mrche de son intervention sur les efforts constatés à la digue de Naples - intervention abordée par l'auteur en fin de matinée et reprise, en fait, au début de l'après-midi, mais regroupée cidessus pour plus de clarté - M. le président insiste sur le fait qu'il croit être le résnmé de cotte interven- 
Lion: la surélévation constatée intéresse seulement la moyenne entre les deux niveaux extrêmes atteints par la houle sur la jetée.

M. le Président Larras signale qu'une autre raison de T'aecident de Gênes lui a semblé pouvoir se dégager de la réunion de la matinée: les ouvrages reposant sur ut bloc de garde, les cotes de fond sont: $-0 \mathrm{~m}$ et $-10 \mathrm{~m}$, au lieu de $-10,50 \mathrm{~m}$ et $-11,50 \mathrm{~m}$; l'amplitude de la houle ayant été estimée à $7 \mathrm{~m}$, les conditions de déferlements, évaluées à une fois et demie l'amplitude, au Congrès de Bruxelles, et à deux fois l'amplitude, au Congrès de Rome, se trouvaient donc Jargement remplies.

M. LaYal croit se souvenir que la surélévation est d'autant plus importante que la cambrure est grande et, d'autre part, que le rapport de la longueur d'onde à la profondeur est également fort. Or, à Naples, ces grandeurs étaient faibles et l'application des formules au second degré de M. Mrche ne lui paraissait pas rendre compte de la surélévation telle qu'elle a été constatée : les conditions n'étaient pas celles correspondant d'après to calcul, à une très forte surélévation, et celle-ci s'est produrte quand même. De toutes façons, M. LAYAL. indique à M. Miche qu'il lui communiquera les chiffres réels des observations de Naples, aux fins d'analyse.

M. Bavderarre demande à $M$. le Président Larras si les informations acquises à ce jour, en ce qui concerne le déplacement vers le haut du point moyen des oscil. lations au droit du parement d'une digue verticale, peuvent conduire à réviser les indications admises relalivement à l'amplitude de la houle au large, lors de la tempète du 3 février 1934 à Alger.

M. Lanras indique, en réponse à la question poséc par H. Baudelarre, que les mesures de houle de la tempête du 3 février 1934 à Alger ont été des mesures directes par observation visuelle ou par cinématographie de la surface des lames et des mouvements de bouées, et qu'on r'a utilisé la méthode de la surélévation du « niveau moyen $\gg$ qu'à titre de contrôle accessoire de ces mesures.

M. DE Rouville estime, à la faveur des nouvelles informations dues aux IVes Journées, qu'il serait intéressant de revoir ou de mieux établir une relation entre la longueur d'onde, lamplitude de la houle, etc; au point de vue notamment de la surélévation du niveau moyen, relation qui devrait tenir compte de la cambrure, indiquée comme ayant une notable influence.

Il demande, d'autre part, si, à la favenr de certains rapports, il n'y aurait pas lieu de vérifier à nouveau le bien-fondé des formules produites au Congrès de Navigation de Rome, sur le talus des digues en enrochements.

Il rappelle, enfin, que l'une des causes essentielles de l'accident d'Alger (février 1934) fut l'affouillement déterminé au pied du massif d'enrochements par la vitesse de l'eau, du fait du fort clapotis, affouillement dans lequel glissèrent les enrochements et les blocs de la muraille verticale précipités, on le sait, du côté du large.

En réponse à M. Mrche, M. le Président Larnas indique que, dans la tempête du 3 février 1934 à Alger, la houle régulière était très loin des conditions de déferlement, par suite de sa cambrure, mais aussi parce que la mer passait par-dessus le nouvean couronnement de la jetée du Nord, jeté à talus doux, qui venait d'être fait à $7,50 \mathrm{~m}$ quelques mois avant, exactement comme s'il n'y avait pas eu de jetée.

M. DE Rouville remarque que, la houle étant trềs longue, la cambrure n'était pas tellement forte.

En conclusion, M. Baudelaire rappelle qu'à la suite de l'accident du port d'Alger, la décision a été prise de faire des digues à talus. Il confirme, d'après les rapports d'observation et des mesures faites entre temps, la relation de cet accident telle qu'elle a été donnée par
M. DE Rouvulte, c'est-á-dire : la création d'une souille en avant de l'ouvrage, la disparition des enrochements du soubassement dans cette souille et l'effondrement de la digue.

M. Cavenex n'a rien d'important à ajouter à son rapport tel qu'il a été publié, si ce n'est pour confirmer que les deux défauts de la digue de Gênes (partie en blocs) résident: d'une part, dans le manque de liaison des blocs et, d'autre part, dans l'insuffisance de la cote de repos des bloes ou, si l'on préfère, de la partie supérieure des enrochements.

La position trop haute de ceux-ci a entraîné une surélévation du niveau moyen, ainsi que du niveau maximum, entraînant des pressions très importantes sur le mur vertical. Celles-ci ont, alors, dépassé la résistance au glissement des blocs. La destruction a commencé par le départ vers l'intérieur de la série supérieure et le phénomène s'est poursuivi jusqu’à la $3^{2}$ assise, c'est-à-dire jusqu'au moment où la poussée est devenue inférieure à cette résistance au glissement.

M. Cavener pense, en conclusion, que les digues å parois verticales ne doivent pas être définitivement condamnées du fait de l'accident de Gênes, mais qu'au contraire, en prenant des caractéristiques suffisantes, comme celles résumées dans son rapport, on peut continuer de construire de tels onvrages. (Voir plus haut (séance du matin) l'intervention de M. Dhaille sur le rapport de M. Cavenel.)

M. DE Rouville estime que la possibilité de maintenir les digues verticales est interressante, notamment par de grandes profondeurs qui rendent prohibitives les digues $\grave{a}$ talus. Mais les digues rerticales étant également très chères dans ces cas-là, parce qu'il faut leur donner une grande largeur, M. DE Rouville pense qu'il faudrait trouver un système pour que cette surlargeur ne coûte pas trop cher.

M. Cavenel estime que cela dépend aussi des disponibilités en enrochements et de leur prix de revient.

Comme suite à la suggestion de M. DE RouviLLE sur le calcul des digues verticales, M. Laval croit que les efforts globaux qui s'exercent effectivement sur l'ensemble de la muraille sont largement couverts par ceux qui résultent du calcul suivant les règles fixées, par cxemple, au dernier Congrès de la Navigation. Mais, par contre, il pense qu'il peut $\mathrm{y}$ avoir une distribution verticale de ces efforts globaux, differente de celle qui est envisagée généralement dans la théorie hydrostatique et, en particulier, il y aurait un renforcement des efforts au niveau du point moyen, où plus exactement, au niveau du plan de repos de la mer et au-dessus, qui pourrait expliquer certaines destructions par glissement des assises supérieures des digues sans qque, cependant, la fondation soit touchée.

M. Laval signale avoir été frappé par le fait qu'une digue verticale n'est pas parfaitement réflèchissante spécialement pour les houles complexes, comme le sont les houles naturelles, et qu'une certaine énergie peut donc agir sur la superstructure et créer certaines concentrations d'efforts. Cela, d'ailleurs, est tout à fait conforme a la pratique de construction des digues verticales ou tout en calculant les blocs supérieurs au glissement, on a reconnu la nécessité d'ajouter des liaisons entre les blocs parce qu'il peut y avoir des percussions locales. Il $y$ aurait donc une distinction à faire entre la stabilite globale de l'ouvrage et les efforts Iocaux; cette question pourra être reprise à propos de l'examen du rapport de M. Hensen. M. Cavenel partage entièrement eette conception.

M. le Président remercie M. Cavenel des renseignements précieux qu'il nous a apportés de Gênes et de l'analyse, à laquelle il s'est livré, de ces renseignements : cette analyse, d'une part, confrme un grand nombre de points que l'on connaissait et qu'on avait étudiés, et, 
d'autre part, précise la portée et les limites de certaines de ces règles.

M. Cavener à tenn à ajouter qu'il a été aidé, dans son étude par M. l'Ingénieur VEx.

M. Ie Président se sert de l'hommage rendu par M. Cayenel à M. Vey comme transition pour passer à la. question suivante, qui concerne le rapport de M. l'Ingénieur en Chef Couteaud, représenté par M. l'Ingénieur Vey.

M. VEy croit devoir préciser que les études théorique et expérimentale faites sur la digue verticale de Marseille font apparaitre dans la stabilite de cet ouvage une marge de sécurité qui a été évaluée à $30 \%$ et qui reprësente l'écart entre, d'une part, les houles régulières maxima susceptibles d'attaquer réellenent l'ouvrage et déterminées d'après les observations et les prévisions theoriques et, d'autre part, les houles d'etude limites au-dessus desquelles la stabilite vérifiee par les calculs et par les essais hydrauliques ne se trouverait plus assurée. Cette marge est destinée à tenir compte de différents éléments, en particulier de l'incertitude qui pèse sur la détermination des houles maxima régulières auxquelles pourra être réllement exposé l'ouvrage et de l'action des houles irrégulières dont les effets ne sont pas encore bien connus.

Il est en effet bien établi qu'à Gênes ce sont les déferlements très importants causés par des houles irrégulières et favorisés par la faible hauteux de la muraille verticale qui ont contribue à provoquer la rupture sur $450 \mathrm{ml}$ de la partie de la digue la plus récente et considérée comme la plus résistante et qui ont aggravé les destructions subies par toute la digue extérieure.

M. VEY propose donc que les études théorique ef expérimentale des ingénieurs spécialisés et des laboratoires hydrauliques soient poursuivies sur les houles irrégulières en vue de définùr:

a) les conditions de leur fomation et de leur évolution,

b) les conditions de \& brisure $\$$ sur les ouvrages,

c) les effets exercés sur les superstructures.

M. VEy rappelle les améliorations à l'étude dans la digue verticale du port de Marseille. Ces améluorations qui sont visees au rapport $n^{\circ} 11$ ont pour objet la consolidation de la risberme en blocs naturels de la plateforme de fondation de la muraille et la protection des joints des maçonneries des massifs monolithicques conslituaut cette inuraille. Les premiers résultats constatés depuis la date d'cnvol du rapport sont satisfaisants.

M. Miche demande a M. VEr comment la hauteur maximum de houle à Marseille $(5,40 \mathrm{~m})$ a été déterminée. Si elle résulte d'une différence de niveau de $2 \times 5,40=10,80 \mathrm{~m}$ observée sur la digue, il est possible que la houle incidente ait été plus forte que $5,40 \mathrm{~m}$. En effel, la reflexion d'une jetee verticale n'est jamais totale, sauf lorsque la brisure du clapotis s'amorce, auquel cas, au contraire, la dénivellation totale sur la digue pourrait excèder le double de celle de la houle incidente. Pour le caleul d'une digue verticale, on peut admettre, par convention, une réflexion totale, c'esta-dire assimiler à $4 h$ la dénivellation observée, et dans ce cas, Ia question posée importe peu. Elle offe, par contre, de l'intérêt lorsqu'il s'agit d'estimer la hauteur maximum d'une houle incidente à un emplacement déterminé, en vue de la comparer, par exemple, a celles obtenues allleurs par des méthodes directes (visées sur des bouées, etc.).

M. VEY répond que le procédé utilisé par le port de Marseille pour la mesare de l'amplitude des houles est basé :

a) Sur la lecture de l'affleurement maximum du clapotis sur une échelle installée contre la muraille verticale de la digue du large; b) Sur la relation existant aux différents niveaux de la mer, entre cet affleurement et l'amplitude do la houle. Cette relation est donnce par des abacues qui ont été établis après essais hydrauliques.

Il est certain que la valeur des résultats obtenus dépend, essentiellement des conditions suivantes :

- Attaque normale de la digue verticale ou sous an angle assez faible qui parait devoir être limite entre 30 et $40^{\circ}$.

- Formation d'un clapotis quasi normal.

A Marseille, les observations portent uniquement sur les houles du secteur Sud-Onest-Onest qui sont les plus violentes et les plus dangereuses. Comme ces houles sont généralement assez régulières et qu'elles attaquent normalement la muraille de la digue qui comporte une grande hauteur immergée, on admet que le clapolis qu'elles engendrent se rapproche de celui donné sur modèle réduit par les houles pures essayées en laboratoire.

Les essais hydrauliques effectués pour la mise au point des abaques ont d'ailleurs démontré, d'une part que le rapport des amplitudes des houles réflechies et incidentes ne dépassait jamais $90 \%$ et pouvait mème descendre bien au-dessous pour les grandes longueurs d'onde et, d'autre part, que l'amplitude totale mesurée sur la paroi verticale était dans tous les cas inférieure a celle donnée par les formules théoriques.

M. VEY jndique qu'il y aurait intérêt :

- A ce que les ports qui utilisent des procédés de mesures analogues fassent connaître :

a) leur avis sur les résultats obtenus,

b) les améliorations susceptibles d'y être apportées,

c) éventuellement la comparaison faite entre ces résultats et ceux donnés par d'autres procédés (enregistreur, simple bouée, etc.),

- A ce que les ports voisins soumis aux houles maxima de même direction échangent annuellement leurs observations.

A cet égard un échange d'informations a été decidé entre les ports de Gênes et de Marseille.

M. le Président remercie M. VEY de son exposé complémentaire et en retient notamment l'intérêt des essais en cours pour la consolidation des soubassements de fondation des murailles verticales et pour la protection des blocs monolithiques par l'emploi du bitume.

An sujet des houles irrégulières, dont vient de parler M VEx, M. Cavenex pense qu'elles pourraient provenir de résurgences de tempêtes lointaines (1.000 ou $1.500 \mathrm{~km}$ ), qui arwiveraient à passer et seraient très chargées en énergie.

11 eite à l'appui de cette opinion le fait que, la cause originelle de la houle étant le vent, il $n^{\prime} y$ avait pas plus à Nice qu’à Gênes de vent local quelques henres avant la tempête qui a causé les dégats qu'il vient d'exposer.

Il faudrait done rechercher les causes de telles houles, dites exceptionnelles on irrégulières.

M. Lavat signale que les théories de M. Mrche sur les trains d'onde peuvent déja apporter quelques éclaircies sur ce problème.

Sur la même question, M. DE Rouville rappelle qu'une simple dépression en déplacement a chance d'engendrer des vents et, par suite, des houles de directions progressivement différentes, qui interferent tout autour de la zone moyenne d'action. Ce cas très fréquent, comporte, a sa connaissance, quelques exceptions quand la disposition et la progression du groupe des isobares maintiennent longtemps les vents daus une même direction, ce qui donne lieu à des houles très fortes avec des 
vents encore moyens; c'est ce qui est arrivé, sans doute, pour la dépression, se déplaçant Nord-Sud, qui a causé la catastrophe d'Alger.

Ouant à la suggestion de M. VEx, tendant à voir abais(e) niveau des couronnements de digues verticales, M. IE Rouville estime, au contraire, qu'il ne faut pas laisser la houle réfléchie ou légèrement déferlée se deverser sur le parapet, car, la sous-pression s'exerçant sur le massif vertical, tend à le soulever, comme nos tourelles en mer, trop peu hautes; toute surélévation du massif l'assied davantage sur sa base.

ir. Cavenel se déclare entièrement d'accord avec M. De Rouville.

M. BAUdelaire voudrait savoir comment les ingénieurs au port de Marseille ont déterminé les frais d'entretien des digues a talus, d'une part, et des digues verticales, d'auire part. Il souligne la difficulté qu'il y a, dans celte détermination, à faire le départ entre l'entretien strict et l'amélioration continue des ouvrages. Il semble, d'ailleurs, d'après les chiffres donnés par les ingénieurs du port de Marseille, que la faible différence entre les trais relatifs aux digues à talus et aux digues verticales ne constitue pas un facteur déterminant dans le choix de ces types d'ouyrages.

M. Baudelalre ajoute que des groupes d'études sousmarines (hommes-grenouilles et télévision sous-marine) permettent maintenant de suivre le comportement de ces ouvrages.

A propos d'une remarque de M. Miche sur la rareté des coefficients de réflexion très voisine de l'unité, en laboratoire, M. BIESEr signale une série de mesures de coefficients de réflexion sur une paroi verticale publiées par M. Gneslou dans les proceedings de la $5^{\mathrm{e}}$ « Conférence on Coastal Engineering 》, en 1954.

Ces coefficients sont très rarement inférieurs à $90 \%$ même pour des ondes assez cambrées. Mais il faut signaler" que pour l'obtention de ces résultats, il est nécessaire de faire une correction du second ordre. En effet l'onde incidente et l'onde réfléchie ne peuvent pas être considérées comme superposables dès que leur cambrure n'est plus négligeable M. Breser signale que ees corrections ne sont en général pas faites dans les essais industriels.

M. le Président suggère à MM. Bresel, Laval et Mrahe de se mettre en contact pour rapprocher leurs points de vue sur la question du coefficient de réflexion qui, pour les uns, serait la part d'énergie réfléchie, et pour d'autres, la moyenne de l'énergie incidente et de l'énergic réfléchie.

Pour réduire le coût des digues à talus de grandes dimensions que nécessitent les bas-fonds, M. Laval suggère l'emploi du sable pour constituer la partie inférieure de ces digues, comme cela a été fait à la digue de Valparaiso jusqu'à la cote $(-20)$.

M. Cavenel est de l'avis de M. Laval : on peut faire la base de ces digues jusqu’à la limite de la turbulence c'est-à-dire de $4 h 1 / 2$ environ, en toutes sortes d'éléments, de déchets gros ou petits par exemple, moins chers que le sable. Il faut naturellement attendre que cette base se soit stabilisee avant de commencer la construction de la muraille verticale, c'est ce qu'on a fait avec succès à Marseille, cette attente étant de l'ordre d'une année.

Sur la proposition de M. le Président, la discussion du rapport $n^{\circ} 9$ (M. Lhenmitre) est scindée en deux parties : $1^{\circ}$ Loi de probabilité, ou de répartition, des amplitudes et des périodes de houle.

$2^{\circ}$ Opportunité de construire des ouvrages non infiniment robustes.

Sur le premier point (loi de répartition) M. Bieser demande à M. Miche comment la courbe empirique, obtenue par M. Marié à Casablanca (Rapport n' 4, Ques- tion I), donnant la probabilité cumulee des vagues d'amplitude $2 h$, et de forme exponentielle en $h$, peut être déduite des calculs de $M$. P. Lhermitre sur les probabilités d'existence cumulée de houles données durant un temps $\geqslant t$ (courbe exponentielle en fonction du temps $t$ ).

M. Mrahe confirme les explications données lors de la discussion sur le rapport de M. MaHÉ. Il s'agit d'une analogie conduisant aux mêmes natures d'intégrales, mais portant sur des variables différentes. Pour éviter toute ambiguinté, M. Miche explicite les intégrales applicables au cas de Casablanca. Au surplus, les deux problèmes, bien que d'essence différente, peuvent être reliés de la facon suivante rendant l'analogie intuitive: pour retrouver la courbe de $M$. Makí, on recherchera une loi de distribution, au cours du temps, des écarts-types de trains de houle ẻlémentaires, e'est-à-dire, ì un facteur constant près, de leurs amplitudes moyennes. Dans le cas de M. Lhermitre, (Rapport $n^{\circ} 9$, Question VII), c'est le temps durant lequel sévit une houle donnee dont la loi de distribution est fixée. Sous cette forme, on établit une proportionalité entre les amplitudes et le temps et, comme la distribution gaussienne admise par M. LHERMrTre conduit à une loi exponentielle, en fonction du temps, pour l'existence cumulée de houles données, une distribution gaussienne pour les écarts-types conduira, de même, à une loi exponentielle de probabilité cumulée pour les amplitudes (loi empirique de M. Maré). Plus généralement, l'analogic pourrait être maintenue pour des lois de probabilité non gaussiennes.

A propos de la loi normale de répartition des durées de la houle, posée, a priori, par M. Lhermitre, M. ViguÉ signale qu'ayant étudié des statistiques assez étendues de vent (vitesses mesurées trois fois par jour sur 7 ans), il a trouvé que, $v$ étant la vitesse du vent, $\log v$ obéissait à une loi normale.

Des études vont être faites sur des enregistrements continus, mais il serait, d'ores et déjà, intéressant de signaler le résultat partiel ci-dessus, lequel pourrait permettre de tenter un rapprochement theorique avec les études de houle, le problème de la corrélation entre le vent et la houle étant, comme l'indiquait M. Cavenel, absolument primordial.

M. LAYAL insiste sur le fait que M. Lhermutre a étudié la repartition des intervalles de temps pendant lesquels une houle règne et non la répartition des amplitudes de houle: l'analogie, purement formelle, entre les Iuis statistiques adoptées par M. Lhenumte et par M. MaHE n'enlève rien, comme l'a expliqué M. Miche, à l'originalité de l'idée de $M$. Lhenmtrte qui traite un problème tout différent.

Sur le second point (opportunité de la solidité des ouvrages), M. le Président considère l'absence d'opposition comme un accord complet sur le principe, rejoignant les idées de M. Prot, qu'il ne faut peut-être pas forcer exagérément sur la « solidité 》 des ouvrages. Il oppose le mot « solidité » à « sécurité 》, toute restriction de la sćcurité étant impensable. Quant à la solidité, sa limite inférieure correspond théoriquement au minimum des dépenses totales absorbées par l'ouvrage : amortissement de la construction et frais d'entretien. Cependant, M. Larras recommande, par expérience, de ne pas s'engager, par une construction trop légère, à des frais d'entretien trop lourds, car ces frais risquent d'être réduits pour des raisons budgétaires et leur réduction entraine alors des travaux de renforcement ou de reconstruction qui, d'un seul coup, absorbent - et dépassent même - toutes les économies escomptées.

M. Lhenumre fait, sur l'ensemble de son rapport $\left(1^{\circ}\right.$ et $\left.2^{\circ}\right)$, trois observations :

1. Le calcul est, en fait, un calcul de probabilités com- 
posées, analogue à celui exposé à M. Miche, à qui il doit, d'ailleurs, la suggestion du mode de calcul.

2. La loi normale adoptée ne l'a été qu'à titre d'exemple; il est bien évident qu'il peut y avoir intérêt a adapter le mode de calcul à d'autres lois de probábilité, telles que les courbes de Pierson ici proposées par M. l'Ingénieur en Chef Larras. Toutefois, les calculs ne sont pas aussi simples.

3. La méthode proposée présente, outre l'intérêt souligné par MM. le Président et le Rapporteur Général, lavantage de traduire en termes, à son avis, plus parlants, le choix de la houle à prendre en considération, qui se transforme en probabilités de ruine. Enfin, les deux courbes théoriques proposées figure 4, peuvent schématiser, par exemple, les types de brise-lames, longuement évoqués précédemment, de la jetée verticale, dont la stabilité disparait brutalement pour de faibles dfférences de l'amplitude, et de la jetée en enrochement dont la courbe de stabilité serait plutôt du type II.

Le rapport de M. Hensen ne peut ctre discuté, par suite de l'absence de son auteur qui, malnde, a do rejoindre son pays.

Invité par M. le Président, M. Laval ajoute, à titre général, que la question du coefficient de réflexion reste une question importante : il sera très souhaitable de bien poser le problème, ainsi que M. Bieser l'a fait ressorlir, de réunir beaucoup de données expérimentales et, enfin, de savoir s'il y a des dissipations d'énergie et ce que devient l'énergie dissipée dans la réflexion.

M. le Président remercie les participants et les assistants à la discussion et félicite M. Laval du très important travail qu'il a fourni, malgré ses nombreuses occupations.

\section{LES ÉNERGIES DE LA MER}

Compte rendu intégral des QUATRIÈMES JOURNÉES DE L'HYDRAULIQUE. de la Société Hydrotechnique de France

Deux volumes, brochés........... $8000 \mathrm{~F}$ 